\title{
STUDY ON PLANTAR PRESSURE OF PEOPLE IN DIFFERENT AGE During Level Walking
}

\author{
Gang Qiao \\ ${ }^{1}$ School of Mechanical Engineering, Zhengzhou University of Industrial And Technology, 451100, China \\ qg523612026@163.com
}

\begin{abstract}
To investigate the influence of age on plantar pressure in normal people during level walking, ground reaction force data from 20 normal people of different age were collected by the employment of Three-Dimensional force platform system, and the force data in anterior-posterior, medio-lateral and vertical directions were normalized and transformed into force-time curves. The characteristic parameters were selected and analyzed by using statistical methods. The plantar pressure in normal people of different age was comparatively analyzed. Results show that a certain degree of difference was found in the plantar pressure distribution in normal people of different age during level walking: for the old group, the time parameters of gait in vertical and anterior-posterior direction came earlier than those in the young group. It may be due to the fact that the step length of the old person is smaller than that of the young person and the center of gravity of the body crossing the hip appears earlier in the old group than in the young group.
\end{abstract}

Keywords: plantar pressure; natural level walking; age

\section{Introduction}

Normal walking is a fairly mundane activity in our daily life, which is especially important to the elderly people.However, with the growth of age, instability occurs during level walking.Previous studies have shown that about 53\% of the elderly fall is caused by the instability of walking or standing, and the fall will cause other diseases which could bring great pain to the elderly.Consequently, it is quite significant to investigate the plantar pressure of people in different age during level walking. This paper, from the perspective of the plantar pressure distribution, collects the sample of force data and make comparative analysis on the effect of age to the plantar pressure during natural walking of human.

\section{Experimental method}

\subsection{Test object}

The subjects were divided into youth group and elderly group according to their age. Each group consisted of 5 male and 5 female subjects. The average age of the young group was 20 years and the average age was 22 years, with an average height of $175.7 \pm 2.3 \mathrm{~cm}$ and an average body weight of $65.8 \pm 2.1 \mathrm{~kg}$. The age group was 61-65 years old with an average age of 63 years Was $172.3 \pm 1$. $7 \mathrm{~cm}$ and the average body weight was $71.8 \pm 1.4 \mathrm{~kg}$. All testers are adults who are in good health, have normal behavior, and have no history of the motor system.Before the experiment, the experime nter was informed of the purpose of the experiment and the experimental program, all subjects were informed and agreed to this experiment, voluntarily participate in this experiment.

\subsection{Experimental method}

\subsubsection{Experimental environment}

The experimental corridor consists of a trail and a dynamometer platform in which the dynamometer platform is placed in the center of the experimental corridor, and the platform is placed with a high flush track with a measuring platform equal to $3800 \mathrm{~mm} \times 600 \mathrm{~mm} \times 60 \mathrm{~mm}$. The experimental corridor is shown in figure 1 . The experimental equipment mainly includes the computer, the three-dimensional force measuring platform system and so on. 
In the experiment, the pressure data acquisition of human body is based on the JP6060 type three dimensional force measuring system, which is composed of the force-measuring platform, the signal processing system and the data analysis system. The size of the force-measuring platform is $600 \mathrm{~mm}$ $\times 600 \mathrm{~mm} \times 60 \mathrm{~mm}$, the sampling frequency is $300 \mathrm{~Hz}$, the static detection error is less than $0.5 \%$, and the detection range can be adjusted according to the actual needs. Signal processing system and data analysis system can realize real-time data processing.

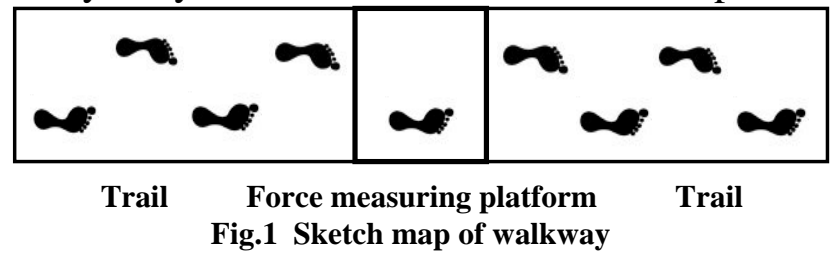

\subsubsection{Experimental process}

The tester walked barefoot through a normal gait. During the experiment, the subjects in their usual walking speed from the start end side trails begin walking through mechanical testing platform, walk to the other side of the trail through the distal, force measuring platform, to ensure complete on one foot on the table to get the complete data of plantar pressure of the left or right. In order to ensure stable and reliable experimental data, before the formal test, the test requirements in accordance with the normal requirements of the experiment to practice 15 minutes to enter the state, after practice began the formal experiment. After the start of the experiment, we tested the left foot and the right foot for each test 10 times, the data obtained from the 10 tests can be considered as a stable and reliable data. The researchers recorded the 10 left foot data and the 10 right foot data into the database for statistical analysis.

\subsection{Data processing method}

The force can be measured in the process of walking in three direction of the ground reaction force, that is $\mathrm{Y}$ (antero-posterior direction), $\mathrm{X}$ (medio-lateral direction) and $\mathrm{Z}$ (vertical direction). In order to eliminate the differences between individual participants, three direction of the ground reaction force data were measured and the data were normalized to the weight of the testers after the mechanical test, the single support phase was normalized to one foot time after the test data. The single foot single support phase refers to the time from the left foot or the right foot of the heel contact force measuring platform until the toe toe off the measuring platform.

Mechanical data and time data on three directions after normalization can be obtained by fitting the test of plantar pressure force time curves (Fig. 2), the relative value of $\mathrm{X}$ axis represents time of this graph, which is $\mathrm{t} / \mathrm{Ts}$ (Ts is the single foot single support phase), $\mathrm{Y}$ axis represents the relative value of pressure that is, $\mathrm{F} / \mathrm{W}$ (W-weight of tester).

We extracted the following 17 characteristics as gait parameters were statistically analyzed, 7 of which feature extraction and direction of the force time curve, 4 feature extraction about the direction of the force time curve, 6 feature extraction in vertical direction of the force time curve, the amount of each feature meaning Figure 2 shows. According to the obtained data, the 10 left foot data of the above 17 parameters and the data of the right foot of the above 10 parameters are taken as the analysis samples. We use the method of $\mathrm{T}$ test to analyze the differences between 17 feature of the young group and the old group, for each feature, the matching test of the young group and the old group of data, the return value is $\mathrm{P}$, when $\mathrm{P}<0.05$, that there is a significant difference between the plantar pressure of the young group and elderly group distribution, when $\mathrm{P}>0.05$, illustrate the plantar pressure distribution of the young group and the elderly group had no significant difference

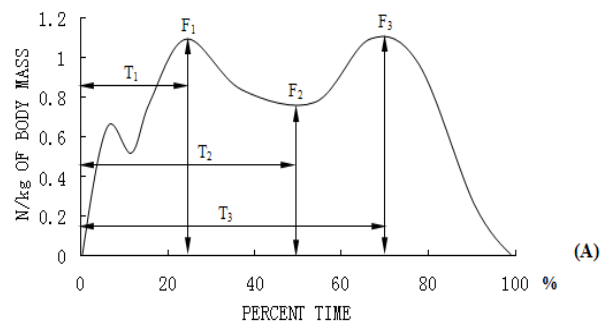

(a)force in vertical direction

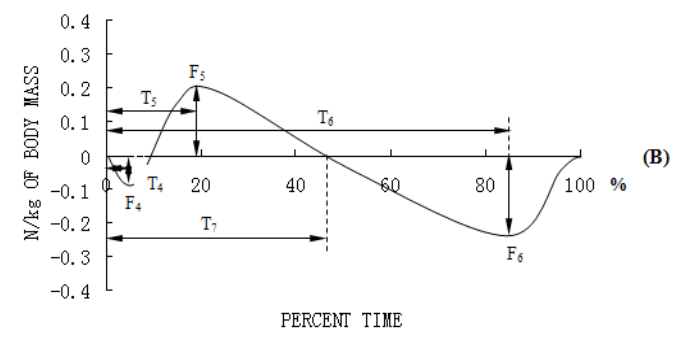

(b)force in antero-posterior direction 


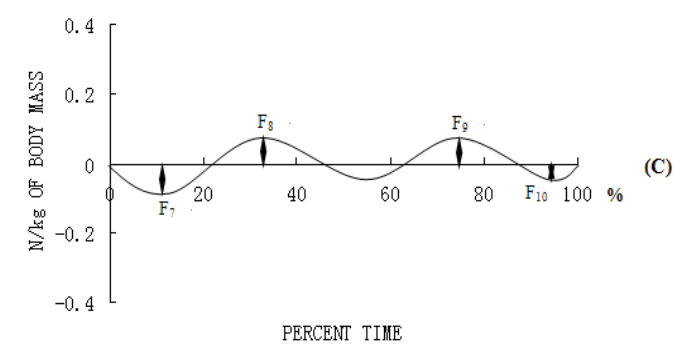

(c)force in medio-lateral direction

Fig.2 The three dimensional ground reaction forces in human during level walking

\section{Experimental results and data analysis}

\subsection{Experimental results}

The results obtained from the plantar pressure data of the youth group and the elderly group were shown from table 1 to table 3

Tab.1 The statistic results of the six parameters from vertical force-time curve

\begin{tabular}{|c|l|l|l|l|}
\hline \multirow{2}{*}{ Characteristics } & \multicolumn{2}{|c|}{ Youth Group } & \multicolumn{2}{c|}{ Elderly Group } \\
\cline { 2 - 5 } & Left & Right & Left & Right \\
\hline $\mathrm{F} 1$ (B.W.) & $1.08 \pm 0.12$ & $1.07 \pm 0.07$ & $1.05 \pm 0.08$ & $1.06 \pm 0.23$ \\
\hline $\mathrm{F}_{2}$ (B.W.) & $0.86 \pm 0.07$ & $0.85 \pm 0.12$ & $0.84 \pm 0.19$ & $0.84 \pm 0.32$ \\
\hline $\mathrm{F}_{3}$ (B.W.) & $1.11 \pm 0.25$ & $1.12 \pm 0.28$ & $1.09 \pm 0.31$ & $1.08 \pm 0.43$ \\
\hline $\mathrm{T}_{1}$ (\%) & $25.4 \pm 1.6$ & $25.8 \pm 3.6$ & $24.2 \pm 2.3$ & $25.3 \pm 1.6$ \\
\hline $\mathrm{T}_{2}$ (\%) & $46.7 \pm 2.1$ & $45.9 \pm 1.3$ & $45.1 \pm 1.8$ & $44.2 \pm 2.1$ \\
\hline $\mathrm{T}_{3}$ (\%) & $75.2 \pm 3.5$ & $76.5 \pm 3.1$ & $72.8 \pm 2.7$ & $74.5 \pm 3.9^{\mathbf{4}}$ \\
\hline
\end{tabular}

Tab.2 The statistic results of the seven parameters from antero-posterior force-time curve

\begin{tabular}{|c|l|l|l|l|}
\hline \multirow{2}{*}{ Characteristics } & \multicolumn{2}{|c|}{ Youth Group } & \multicolumn{2}{c|}{ Elderly Group } \\
\cline { 2 - 5 } & \multicolumn{1}{|c|}{ Left } & \multicolumn{1}{c|}{ Right } & Left & Right \\
\hline $\mathrm{F}_{4}$ (B.W.) & $-0.08 \pm 0.02$ & $-0.07 \pm 0.09$ & $-0.07 \pm 0.03$ & $-0.07 \pm 0.10$ \\
\hline $\mathrm{F}_{6}$ (B.W.) & $-0.23 \pm 0.02$ & $-0.22 \pm 0.11$ & $-0.24 \pm 0.05$ & $-0.23 \pm 0.08$ \\
\hline $\mathrm{T}_{4}(\%)$ & $4.6 \pm 1.2$ & $4.5 \pm 2.1$ & $4.2 \pm 0.8$ & $4.3 \pm 1.4$ \\
\hline $\mathrm{T}_{5}(\%)$ & $18.3 \pm 1.4$ & $18.0 \pm 0.5$ & $17.8 \pm 1.7$ & $17.6 \pm 2.3$ \\
\hline $\mathrm{T}_{6}(\%)$ & $84.1 \pm 2.1$ & $84.3 \pm 1.6$ & $83.2 \pm 1.4$ & $83.8 \pm 3.2$ \\
\hline $\mathrm{T}_{7}(\%)$ & $45.0 \pm 2.6$ & $44.8 \pm 0.9$ & $43.2 \pm 1.4$ & $44.6 \pm 2.7^{\mathbf{4}}$ \\
\hline
\end{tabular}

Tab.3 The statistic results of the four parameters from medio-lateral force-time curve

\subsection{Data analysis}

\begin{tabular}{|c|l|l|l|l|}
\hline \multirow{2}{*}{ Characteristics } & \multicolumn{2}{|c|}{ Youth Group } & \multicolumn{2}{c|}{ Elderly Group } \\
\cline { 2 - 5 } & \multicolumn{1}{|c|}{ Left } & \multicolumn{1}{c|}{ Right } & \multicolumn{1}{c|}{ Left } & Right \\
\hline $\mathrm{F}_{7}$ (B.W.) & $-0.05 \pm 0.13$ & $-0.05 \pm 0.08$ & $-0.08 \pm 0.16$ & $-0.08 \pm 0.04$ \\
\hline $\mathrm{F}_{8}$ (B.W.) & $0.06 \pm 0.17$ & $0.07 \pm 0.12$ & $0.07 \pm 0.14$ & $0.08 \pm 0.21$ \\
\hline $\mathrm{F}_{9}$ (B.W.) & $0.05 \pm 0.12$ & $0.05 \pm 0.24$ & $0.06 \pm 0.08$ & $0.07 \pm 0.32$ \\
\hline $\mathrm{F}_{10}$ (B.W.) & $-0.03 \pm 0.04$ & $-0.03 \pm 0.18$ & $-0.04 \pm 0.13$ & $-0.04 \pm 0.07$ \\
\hline
\end{tabular}

\subsubsection{Vertical direction}

The component of vertical direction is the main component of plantar pressure. During normal walking, the heel to heel is called a gait cycle. A gait cycle can be divided into 6 turning points. Take the left lower limb as an example: the left foot follows the ground, the left foot is flat, the left toe is off the ground, the right foot follows the ground, the right foot is flat, and the right toe to the ground is first-6 turning points. In a complete gait cycle, there is in every turning point has a maximum force in $\mathrm{Z}$ direction, one side heel strike when there is a large value of $\mathrm{F}$, with the flat side of the foot, the stress decreases gradually, until the foot side completely normally, by a minimum force reached F2 then, the lateral heel off the ground, a maximum value of F3 reached to the side and toe off, it can be seen that the force-time curve of the vertical direction has a typical characteristics of two-peaks. The maximum peak value of the left and right foot curve was about 1.1 times of body weight, and the minimum peak value was about 0.85 times of body weight.

3.2.2 Antero-posterior direction

The force-time curve in antero-posterior direction is basically symmetrical,and there is a maximum of positive value and two minimum of negative value, and the maximum of positive value is about 0.17 
times of the weight, appeared in the $18 \%$ of the single support phase; the two minimum of negative value are about 0.07 and 0.23 times of the weight, appeared respectively in the $4 \%$ and $84 \%$ of the single support phase.

3.2.3 Medio-lateral direction

The medio-lateral direction takes the right of walking as positive,and the force-time curve is basically symmetrical and has s small value, the maximum of the force is about 0.07 times of the weight.

The preceding analysis shows that the GRF value of the vertical direction is larger,while the antero-posterior and medio-lateral direction are smaller when the testers walk normally on the dynamometer.The force-time curve of the vertical direction has a typical characteristics of two-peaks while the curves in antero-posterior and medio-lateral direction are symmetrical about the time axis.

\subsubsection{Difference Analysis}

The T-Test method was used to analyze the differences of gait characteristics of the young group and the elderly group in three directions during the course of natural walking, and the statistical results were shown in table 1 to table3.The results shown that, there was a significant difference at the $\mathrm{T}_{1}$ and $\mathrm{T}_{3}$ in the vertical direction and $\mathrm{T}_{7}$ in the antero-posterior direction during the natural walking of the young group and the elderly group in the plantar pressure distribution, whether left or right; in the vertical direction, the data of $\mathrm{F} 1, \mathrm{~F}_{2}$ and $\mathrm{F}_{3}$ of the elderly group were smaller than those of the young group,consider the possibility of slower pace with the elderly than young people, for gait time parameters $T_{1}, T_{2}$ and $T_{3}$, the data of the youth samples are always larger than those of the elderly group, which may be due to the larger walking distance of young people. In medio-lateral direction, the gait mechanics parameters of $F_{1}, F_{2}$ and $F_{3}$ of the elderly group were larger than those of the young group,as the medio-lateral direction of the force reflects the stability of walking,therefore,it shows the stability of young people during walking were better than the elderly.

\section{Conclusions}

There is no significant difference in the distribution of plantar pressure between the healthy elderly and the youth at normal speed,while the time when the center of gravity passing hip joint was earlier in elderly than the youth,which makes the gait time parameters were smaller in elderly than the youth.

\section{References}

[1] Antonelh Caselli,et a1.The forefoot-to-rearfoot plantar pressure ratio is increased in severe diabetic neuropathy and can predict foot ulceration[J]. Diabetes Care 2002,25:1066-1071

[2] Eleftherios Kellis.Plantar preasure distribution during barefoot standing,walking and landing in preschool boys[J].Gait and Posture 2001,14:92-97.

[3] Joseph Hamill, Mcniven. Reliability of Selected Ground Reaction Force Parameters During Walking[J].Human Movement Science, 1990, 9(2):117-131.

[4] White R , Agouris I , Selbie RD , et al . The variability of force platform data in normal and cerebral palsy gait [J]. Clinical Biomechanics,1999,14(3):185-192.

[5] Charnley J, Pusso R. The recording and the analysis of gait in relation to the surgery of the hip joint[J]. Clinic Orthop,1968,58:1.

[6] Simpson KJ ,Jiang P. Foot landing position during gait influences ground reaction forces[J].Clinical Biomechanics,1999,14(6):396 - 402. 